\title{
Cutaneous Metastasis from Breast Tumour Seeding in Percutaneous Needle Biopsy
}

\author{
Imma Savarese ${ }^{a}$ Federica Scarfi $^{a}$ Alessia Gori ${ }^{a}$ Federica Papi ${ }^{a}$ Antonietta D’Errico ${ }^{a}$ \\ Marta Grazzini ${ }^{a}$ Piero Covarelli ${ }^{b}$ Vincenzo De Giorgi ${ }^{a}$
}

a Department of Dermatology, University of Florence, Florence, Italy;

${ }^{b}$ Department of Surgery, University of Perugia, Perugia, Italy

Dear Editor,

A 79-year-old woman complained of an elevated lesion localized on the neck. 9 years earlier she had been treated with curative surgery and irradiation (QUART), followed by chemotherapy and hormone therapy for an infiltrating ductal carcinoma (G3) with positive stain for estrogen receptor (ER, 100\%), progesterone receptor (PR, 70\%) and Ki-67 (18\%). The skin lesion was characterized as firm, normal skin coloured and painless; it was $2.5 \mathrm{~cm}$ in diameter and sited in the clavicular fossa (fig. 1). Given that the lesion was localized to the access site of a previous percutaneous needle biopsy (PNB) for breast cancer lymph node metastases approximately 12 months previously, the general practitioner postulated the diagnosis of a keloid scar. PNB of the supraclavicular lymph nodes was performed using a co-axial technique with a dedicated needle AG (21G). Fluorescence in situ hybridization (FISH) with dual core probe LSI HER2/neu spectrum orange/CEP 17 spectrum green (PathVision Kit VYSIS) was negative for HER2/neu. The new skin lesion developed rapidly, so it was surgically excised and diagnosed as a subcutaneous metastasis of infiltrating ductal carcinoma, immunoreactive for ER (100\%), PR (90\%) and Ki-67 (25\%).

Cutaneous or subcutaneous tumour seeding after percutaneous procedures are described as a rare complication in literature $[1,2]$. Tumour seeding can result in displacement of neoplastic cells that can initiate tumour growth at a separate anatomic site, along the needle tract. Tumour manifestation occurs as a direct result of this procedure, and should not be confounded with a (more frequently found) local recurrence in which tumour has developed relatively close to the primary tumour, probably due to micro deposits in the surrounding

Fig. 1. Firm and painless skin nodule that appeared a few months after a percutaneous needle biopsy in the clavicular fossa of a 79-year-old woman.

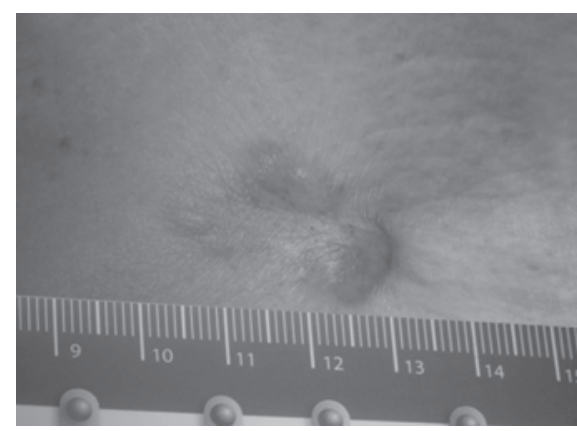

tissues. $0.7-0.9 \%$ of patients with a malignant tumour at any site can develop a cutaneous or subcutaneous metastasis after percutaneous procedures [2]. The duration between the onset of tumour seeding and the previous percutaneous needle biopsy has been shown to vary widely. Clinically, tumour deposits are observed as painful cutaneous or subcutaneous nodules, typically round or oval, with well-defined margins, and are reddish, angiomatous or normal skin coloured. In our patient, the lesion was firm and painless, normal skin colour with irregular margins and mimicked a keloid scar. Generally, histological and immunohistochemical markers for tumour seeding are similar to the primary tumour, as in our case. PNB of lymph node within the neck region, axilla and groin are commonly performed. The potential adverse events of lymph node surgery include lymphedema, numbness, tingling, pain at the site of surgery and difficulty moving the affected body part. Tumour seeding following lymph nodes biopsy, such in this case, is an unusual occurrence. Typically, patients with a history of breast cancer report a tumour seeding directly on the breast region, after a PNB on the primary tumour. Needle tract seeding of malignant cells is detected in $22 \%$ of such patients [1]. In a recently study, a statistically significant difference between seeding occurrence for invasive ductal carcinoma and invasive lobular carcinoma, with rates of $40 \%$ and $15 \%$, respectively, was found [1]. This current case is an unusual presentation of tumour seeding from an invasive ductal carcinoma because the initial tumour was breast cancer lymph node metastases. Any suspicious skin lesion appearing at the site of a PNB must be considered carefully and subjected to cutaneous biopsy to exclude the diagnosis of malignancy.

\section{Disclosure Statement}

The authors have no relevant financial interest in this article.

\section{References}

1 Robertson EG, Baxter G: Tumour seeding following percutaneous needle biopsy: The real story! Clin Radiol 2011;66:1007-1014.

2 Jegou J, Perruzi P, Arav E, Pluot M, Jaussaud R, Remy G: Cutaneous and bone metastases revealing hepatocarcinoma. Gastroenterol Clin Biol 2004;28:804-806.

\section{KARGER}

(c) 2016 S. Karger GmbH, Freiburg 